\title{
Stop! The Polio Vaccination Cessation Game
}

\author{
Scott Barrett
}

\begin{abstract}
Should polio be eradicated worldwide, countries must decide whether to continue to vaccinate with the live-attenuated vaccine, to continue to vaccinate with the alternative, killed vaccine, or to cease vaccinating. To reap a dividend from polio eradication, countries must choose the last option, but vaccination cessation entails interdependent risks as well as rewards. This article models the polio vaccination cessation game, deriving the conditions that support vaccination cessation as an equilibrium, describing the policies and institutions needed to steer countries toward this equilibrium, and presenting crude quantitative estimates of the gains to supporting this equilibrium. JEL classifications: C72, F53, H87, I18, O19
\end{abstract}

The global effort to eradicate polio is an audacious undertaking. It has been supported by every country, inspired millions of volunteers, and been generously funded. It has also made great progress. At the start of the program in 1988, there were 125 polio-endemic countries; today there are 4. Previously, there were more than a thousand new polio cases each day; today, that is the number of people who are paralyzed by polio each year. And, yet, despite these achievements, polio remains a global threat. Eradication was to have been completed by 2000, but from that year onward, and despite numerous tactical adjustments and a redoubling of efforts, the annual number of new polio cases worldwide has varied from around 500 to 2,000, with no evidence of a downward trend. The obstacles to achieving eradication are enormous, but eradication is still considered feasible, and support for the attempt remains strong. Indeed, confidence is so high that the World Health Organization (WHO) is already

Scott Barrett (sb3116@columbia.edu) is the Lenfest-Earth Institute Professor of Natural Resource Economics at Columbia University. The author is grateful to Bruce Aylward, director of the Global Polio Eradication Initiative, for many illuminating discussions, and to Robert Deacon, Radboud Duintjer Tebbens, Harley Feldbaum, Nicholas Grassly, Steve Landry, Jaime de Melo, Mark Pallansch, Kimberly Thompson, Linda Venczel, and three anonymous referees for comments on earlier drafts. Kimberly Thompson and Radboud Duintjer Tebbens also guided the author through their important work on this topic. The Bill \& Melinda Gates Foundation supported this research through the Global Health and Foreign Policy Initiative at the Johns Hopkins University School of Advanced International Studies.

THE WORLD BANK ECONOMIC REVIEW, VOL. 24, NO. 3, pp. 361-385

doi:10.1093/wber/lhq018

Advance Access Publication February 11, 2011

(C) The Author 2011. Published by Oxford University Press on behalf of the International Bank for Reconstruction and Development / THE WORLD BANK. All rights reserved. For permissions, please e-mail: journals.permissions@oup.com. 
looking ahead to what will happen after polio is eradicated. This article is concerned with this post-eradication situation. ${ }^{1}$

Why eradicate? The main reason is to avoid future infections and vaccinations. In the case of smallpox, the only infectious disease to have been eradicated successfully thus far, the value of the avoided infections and vaccinations was enormous. Indeed, the eradication of smallpox may have been the best public investment the world ever made. ${ }^{2}$ For polio, the returns to eradication will be smaller, but they will be positive even for the poorest countries provided that post-eradication risks are kept very low and vaccination stops. ${ }^{3}$ In the case of smallpox, countries could stop vaccinating on their own, independently. ${ }^{4}$ For polio, this approach would be dangerous.

The incentive to stop vaccinating depends on the post-eradication risks. There are many such risks for polio, but the most important is the risk that the genetically unstable, weakened virus strains in the oral polio vaccine (OPV)the main tool for eradicating the three "wild" polioviruses (types 1, 2, and 3) in developing countries-will mutate back and acquire the capability to cause disease and to spread. ${ }^{5}$ Should this happen after the wild polioviruses have been eradicated, eradication will succeed only in substituting vaccine-derived viruses for the wild viruses-a pointless victory if, as seems likely, these circulating vaccine-derived polioviruses (or cVDPVs, as they are known) evolve to resemble the wild viruses. Countries would then need to continue to vaccinate

1. In this article, polio is considered "eradicated" if it is certified as being eradicated. Certification, as determined by the Global Polio Eradication Initiative, requires that all countries maintain excellent surveillance, demonstrate zero transmission of the wild viruses for at least three consecutive years, and reduce the risks of reemergence; for details, see http://www.polioeradication.org/content/fixed/ achievecertification.shtml. The essential point is that "eradication" is a probabilistic concept. For smallpox, the probability of reintroduction is greater than zero, and yet the disease is considered eradicated.

2. By one estimate, from the international financing perspective, the benefit-cost ratio for smallpox eradication was close to 500:1; see Barrett (2007). The greatest challenge for smallpox eradication turned out to be financing. For an analysis of the financing game, see Barrett (2006).

3. The eradication dividend will be smaller for polio than for smallpox for two main reasons. First, only about 1 in 200 people infected with poliovirus show symptoms, and deaths are relatively rare. For smallpox, every infected person shows symptoms, and around a third perish. Second, the post-eradication risks for polio-the subject of this article-are greater. Barrett and Hoel (2007) derive a simple benefit-cost rule from a dynamic model of eradication and find that the dividend for polio eradication is positive even for poor countries. Thompson and Duintjer Tebbens (2007), using a complementary but more detailed approach, obtain a similar qualitative result. However, both analyses assume no post-eradication risks. For an overview of eradication as a goal of health policy, see Miller, Barrett, and Henderson (2006).

4. Indeed, some countries, including the United States, stopped vaccinating years before eradication was certified. The United States stopped routine vaccination for smallpox in 1972, 23 years after the last case of smallpox in the United States and 5 years before the world's last case of endemic smallpox. Smallpox was declared eradicated in 1980.

5. Any of the three viruses can cause polio, and vaccination against one type does not provide immunity to the other types. In most cases, a trivalent vaccine is used, which protects against all three types. 
to guard against cVDPVs, and the returns to eradicating the wild viruses will be lost. With smallpox, the decision to stop vaccinating could be made independently in the post-eradication era because the vaccine did not contain smallpox virus and was genetically stable. For polio the situation is very different.

So far, confirmed cases of cVDPVs have been found in Afghanistan, Burma/ Myanmar, Cambodia, China, Democratic Republic of Congo, Dominican Republic, Egypt, Ethiopia, Guinea, Haiti, India, Indonesia, Madagascar, Niger, Nigeria, the Philippines, Poland, and Somalia. ${ }^{6}$ The Nigerian cVDPVs are illustrative of the problem examined in this article. These cVDPVs are variants of type 2 poliovirus, the wild version of which was eradicated in $1999 .^{7}$ The Nigerian cVDPVs emerged in 2005, a result of Nigeria's continuing to vaccinate with trivalent OPV, which includes a weakened strain of type-2 poliovirus (as well as of types 1 and 3 poliovirus). The Nigerian cVDPVs spread to Niger in 2006 and to Guinea in 2009; they continue to circulate in Nigeria today. ${ }^{8}$

Countries can protect their susceptible populations from cVDPVs by continuing to vaccinate, but if they do so they will lose the gains from eradication. To secure these gains, the Global Polio Eradication Initiative recommends that all countries currently vaccinating with OPV stop doing so simultaneously after eradication has been certified (see Aylward and Cochi (2004) and Aylward, Sutter, and Heymann 2005).

This article explores the incentives for countries to comply with this plan. It models polio vaccination cessation as a game and characterizes its various equilibria. Estimates of parameter values in the literature are used to present both qualitative and quantitative impressions of the challenge. The main conclusion is that vaccination cessation is efficient and can be sustained as an equilibrium (that is, vaccination cessation can be compatible with both the collective and individual interests of states) but that getting to this equilibrium will require a World Health Assembly resolution-a coordinating device.

There is an additional twist to the story. Smallpox was eradicated using a single vaccine; for polio, two vaccines are being used. In addition to OPV, there is a killed or inactivated polio vaccine (IPV). IPV does not pose the same post-eradication risks as OPV, and rich countries have already switched to IPV. They did so, however, not to reduce the transnational externality of continued OPV vaccination in the post-eradication era but to avoid the 1 in 750,000 risk

6. There is some evidence of cVDPVs having circulated previously in Belarus, Pakistan, Peru, Romania, and the Russian Federation; see Kew and others (2005).

7. Type 2 cVDPV outbreaks have also been detected in the Democratic Republic of Congo in 2008-10; in Egypt in the 1980s to 1990s; in Ethiopia in 2008-09; in India in 2009-10; in Madagascar in 2001-02 and again in 2005; and in Somalia in 2008-09. There have also been outbreaks of type 1 and type 3 cVDPVs.

8. Since OPV works against cVDPVs as well as the wild viruses, the main reason the vaccine-derived viruses continue to circulate in Nigeria is poor vaccination coverage. Of course, once vaccination stops worldwide, the pool of susceptible persons will grow rapidly, allowing any cVDPVs that emerge to spread with greater ease. 
of vaccine-associated paralytic polio with OPV (Kew and others 2005). ${ }^{9}$ For most poor countries, substituting IPV for OPV fails a domestic benefit-cost test; in the post-eradication era, poor countries will prefer not to vaccinate over switching to IPV. The plan to stop OPV vaccination thus puts all of the risk on poor countries. And, yet, should these countries comply with the plan, and should the global risks of cVDPVs fall as a consequence, rich countries will be able to claim their share of the eradication dividend by stopping or at least cutting back on IPV vaccination. There are the makings here of a grand bargain. Indeed, it is argued below that eradication of the wild viruses, followed by time-limited substitution of IPV for OPV in poor countries financed by rich countries, creates an incentive for eventual vaccination cessation worldwide, allowing rich and poor countries alike to reap the rewards of eradication and with all countries bearing an equitable share of the risk burden.

The article is organized as follows. Section I presents a toy model of the eradication and post-eradication games to put the contribution of this article in context and to show the important relations the article is seeking to characterize. Section II briefly discusses the post-eradication risks that are not caused by future use of OPV. There are many such risks, and their magnitude will affect national decisions to stop vaccinating. Section III develops the basic model and derives an analytical representation of the probabilities of polio reemergence. Section IV calculates the possible outcomes and equilibria of the post-eradication game. The focus is on the conditions needed to sustain vaccination cessation as an equilibrium and on ways to steer countries toward this efficient equilibrium. Section $\mathrm{V}$ presents some policy implications of the findings.

\section{A Toy Model of the Eradication and Post-Eradication GAMES}

It will help to begin by putting the main subject of this article in context. Consider the situation within a country's borders. Vaccinated individuals reduce transmission, offering a measure of protection to their susceptible fellow citizens. Because of this externality, fewer people will choose to be vaccinated in a competitive equilibrium than in the social optimum-the rationale for public health policy. Vaccination beyond the competitive equilibrium is called control. If a large enough fraction of a population is immunized, herd immunity will protect all susceptible persons within a country. Control at or beyond this "critical level" is called elimination.

If every country eliminates a disease, the pathogen ceases to circulate anywhere, and the disease is then eradicated. Assuming that countries are identical,

9. According to Kew and others (2005), about 250-500 cases of vaccine-associated paralytic polio occur annually worldwide. The problem arises from the genetic instability of the weakened strains in OPV. IPV does not cause paralysis. 
in the "eradication game" shown in figure 1a, each country has a binary choice. It can control the disease or it can eliminate it. As depicted in the figure, each country obtains a higher payoff $(\pi)$ when it controls rather than eliminates the disease unless all other countries eliminate the disease. The payoff to elimination jumps when every other country eliminates the disease because, once the disease is eradicated, there is no longer a need for any country to vaccinate. Eradication is thus an investment. The upfront cost is the loss a country suffers when it eliminates rather than controls the disease. The return on this investment is the increase in the payoff each country receives when it ceases vaccination. Notice that this game has two Nash equilibria (in pure strategies). In one, every country controls but does not eliminate the disease. In the other, the disease is eradicated. Getting to this efficient equilibrium requires international coordination.

The preceding discussion assumes that there is no need to vaccinate once the disease has been eradicated. However, the payoff to vaccination cessation depends on the post-eradication risk of outbreak. If the risk is very low, it will be in each country's self-interest to stop vaccinating in the post-eradication era. If the risk is very high, countries will continue to vaccinate in the post-eradication era-in which case there will be no gain to eradication. For polio, the risk to each country of stopping vaccination depends on whether other countries stop vaccinating. In figure $1 \mathrm{~b}$, these risks are shown to jump if just one country continues to vaccinate, causing the payoff to vaccination cessation to fall. As in the eradication game, there are two equilibria in this stylized vaccination cessation game. In one equilibrium, every country continues to vaccinate. In the other, every country stops vaccinating. In figure $1 \mathrm{~b}$, the second equilibrium is efficient and yields a surplus - the savings associated with vaccination cessation. There is, of course, a relationship between the surpluses shown in these figures. If the surplus shown in figure $1 \mathrm{~b}$ were an annual value, the present value sum of this stream of future savings would equal the surplus due to eradication shown in figure $1 \mathrm{a}$.

Figure 1. Stylized Eradication and Vaccination Cessation Games

a

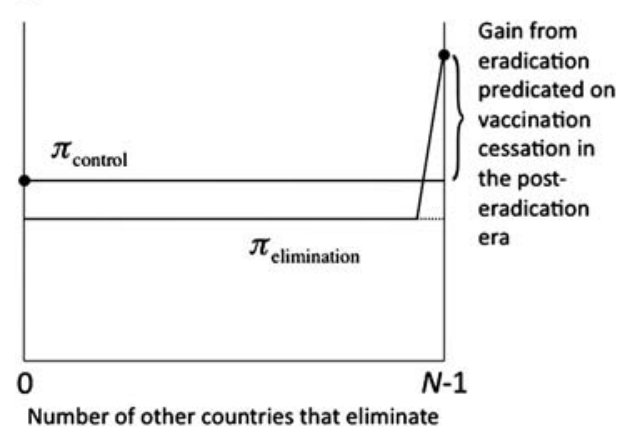

b

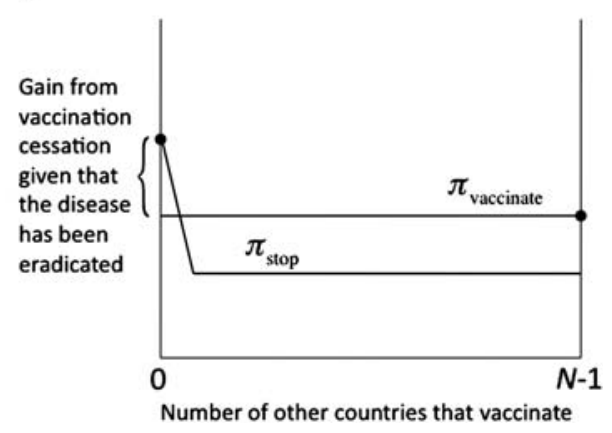


An earlier article modeled eradication as a static game (Barrett 2003; the game depicted in figure $1 \mathrm{a}$ is a cartoon version of that model). ${ }^{10}$ Barrett and Hoel (2007) later modeled the dynamics of the decision to eradicate rather than to control, deriving an expression for the surplus shown in figure $1 \mathrm{a}$, assuming that there were no risks to stopping vaccination in the post-eradication era. The focus in this article is on these post-eradication risks. It derives the payoff curves sketched very crudely in figure $1 \mathrm{~b}$ and uses them to develop a strategy for ensuring that all countries gain from polio eradication.

\section{The Residual Risks of Vaccination Cessation}

The incentive to stop vaccinating depends on the post-eradication risks. ${ }^{11}$ The introduction discussed the risk associated with the continued use of OPV - the main focus of this article. There are, however, other "residual" post-eradication risks.

Some of these will decline over time. For example, vaccine-derived viruses may emerge and circulate in the environment as a legacy of past OPV use. The cVDPVs currently circulating in Nigeria are an example of this risk. Additionally, a very small number of individuals with primary immunodeficiency disorders, when given OPV, may excrete vaccine-derived polioviruses for years (so far, just over 30 such individuals have been identified worldwide). Like the wild viruses, these immunodeficiency-associated vaccine-derived polioviruses (iVDPVs) can set off an epidemic. This risk is also a legacy of past OPV use and will decline over time, after use of OPV has stopped.

Other residual risks can be limited by policy. For example, the risk that wild polioviruses could escape from laboratories that retain samples can be limited by destroying known samples and following strict biocontainment standards for storing retained samples. Similarly, IPV is currently produced from potent wild polio seed stocks, which could escape from a production facility. To reduce this risk, the WHO is seeking to develop IPV manufacturing processes that rely on weakened live poliovirus strains.

These residual risks are taken as given. However, the article explains how the results of the analysis depend on their magnitudes.

\section{The Model}

This section develops a model of the post-eradication game comprising three components-one epidemiological, one probabilistic, and one economic. The game-theoretic model is static; the epidemiological relationships that underpin it are steady-state representations, derived from dynamic epidemiological models.

10. The private and public incentives to eradicate an infectious disease are modeled in Geoffard and Philipson (1997).

11. For a recent overview of the pre- and post-eradication risks, see Barrett (2009). For a detailed assessment of the post-eradication risks, see Duintjer Tebbens and others (2006). 


\section{Epidemiology}

Let $v$ denote the fraction of the population that is immune (from previous exposure to the wild virus or vaccination). The "critical" level of immunization-the level that ensures that incidence of the disease equals zero (and, therefore, that the disease is eliminated)—is given by (Anderson and May 1991)

$$
v^{c}=1-\frac{1}{R_{0}}
$$

Where $R_{0}$ denotes the basic "reproductive number" of the pathogen, or the number of people, on average, who will become infected if one infected person enters a population in which everyone is susceptible. Essentially, $R_{0}$ is a measure of the tendency of a disease to spread (for spread to be possible, $R_{0}$ must exceed 1). For simplicity, assume that this value is identical for all countries, implying that $v^{c}$ will be the same for every country. ${ }^{12}\left(R_{0}\right.$ may be higher in some poor country environments; the sensitivity of the results to variations in $R_{0}$ is examined in section IV.) For polio, $R_{0} \approx 6$. Substituting this value into equation (1) shows that about 80 percent of a population must be immune to eliminate polio within that population. Of course, if polio is to be eradicated, it must be eliminated within every country.

There are $i=1, \ldots, N$ countries. Let $\lambda_{i}$ denote the "force of infection" for country $i$-the probability that a given susceptible individual in country $i$ will become infected, over some short period (the quantitative analysis, presented later in the article, takes this to be one year). Assuming a linear relationship between the force of infection and the gap between the critical and the actual level of immunization (Anderson and May 1991, p. 91) yields

$$
\lambda_{i}=\mu_{i} R_{0}\left(v^{c}-v_{i}\right) \quad \text { for } v^{c} \geq v_{i} ; \quad \lambda_{i}=0 \text { otherwise }
$$

where $\mu_{i}$ is the birth rate for country $i$ (all newborns are susceptible). ${ }^{13}$ Equation (2) implies that a disease can be eliminated $\left(\lambda_{i}=0\right)$ in three ways. First, if the birth rate is zero, the disease will die out on its own. Because polio infection/vaccination confers lifelong immunity, polio can continue to spread only if new susceptible individuals are added to a population. Second, even if the entire population is susceptible (that is, if $v_{i}=0$ ), the disease will not spread if $R_{0} \leq 1$ (letting $v_{i}=0$, equation 2 becomes $\lambda_{\mathrm{i}}=\mu_{i}\left(R_{0}-1\right)$ ). Neither of these

12. The wild and vaccine-derived polio strains are assumed to have the same $R_{0}$. According to Fine and Ritchie (2006, p. 1535), the vaccine-derived viruses "appear to revert towards [the wild poliovirus] phenotype on serial passage in human populations, with strains that have circulated for a year or more showing transmissibility characteristics approaching those of wild viruses." Hence, the assumption appears reasonable. Note that if the basic reproductive number for cVDPVs were less than one, there would be no global risk from continued OPV vaccination. That vaccine-derived viruses have been circulating in Nigeria since 2005 is clear evidence of an $R_{0}$ greater than one for these cVPDVs.

13. Epidemiology models typically assume zero population growth, in which case birth and death rates are equal. That is why, in table $3, \mu_{i}$ is set equal to the inverse of life expectancy. 
conditions applies to polio. To eliminate polio, the third means of elimination must succeed: the vaccination rate must reach the critical level $\left(v_{i}=v^{c}\right)$.

Let polio immunity in country $i$ be given by

$$
v_{i}=v_{i}^{O}+v_{i}^{I}
$$

where $v_{i}^{\mathrm{O}}$ denotes immunization by OPV and $v_{i}^{I}$ immunization by IPV. ${ }^{14}$ These variables are defined in terms of the immunity provided by vaccination. The need for multiple doses to stimulate immunity simply increases the costs of supplying a particular level of immunization (costs are considered later). While equation (3) assumes that either vaccine can stimulate population immunity, it does not assume that the two vaccines have equal efficacy. IPV is believed to be less effective than OPV at reducing transmission. In this model, this handicap is expressed as a higher cost of sustaining population immunity.

Population immunity is a continuous variable. For this analysis, however, it helps to simplify and assume that vaccination is binary. Countries must choose between "routine immunization" (vaccination to the level $v^{c}$ ) using one of the two vaccines and no vaccination. ${ }^{15}$ In the model, this binary choice is represented as follows:

$$
q_{i}^{O} \equiv \frac{v_{i}^{O}}{v^{c}}, q_{i}^{I} \equiv \frac{v_{i}^{I}}{v^{c}}
$$

where $q_{i}^{\mathrm{O}}, q_{i}^{I} \in\{0,1\}$.

\section{Probability of Reemergence}

Imagine now that, after being certified as eradicated, polio is expected to reemerge somewhere in the world with probability $p$ (the probability over an interval of time of at least one outbreak worldwide in the post-eradication era; in the quantitative analysis presented later, the interval is one year). Polio might reappear anywhere and could spread everywhere. This article takes the position that, irrespective of where polio reemerges, it poses an equal threat to every country. ${ }^{16}$

14. Nick Grassly suggested an alternative relation: $v_{i}=1-\left(1-v_{i}^{O}\right)\left(1-v_{i}^{I}\right)$. This multiplicative form implies that countries are better off using a combination of the two vaccines rather than either one on its own. This may be true, but, so far, countries have tended to use one vaccine or the other, not both.

15. This assumption can be relaxed to allow continuous choice; see Barrett (2003).

16. This is an implication of "globalization." When vaccination was stopped in northern Nigeria in 2003, polio spread to 20 polio-free countries. Most of these were in Africa, but the outbreak spread as far as Indonesia. Polio was imported to the United States in 2005 from an unidentified source. As this was a vaccine-derived virus, and the United States and most industrialized countries had stopped using OPV many years before, its source was almost certainly a person vaccinated with OPV in a developing country. Note, however, that spread is not random; neighboring countries are at relatively greater risk. Modeling the spatial dimension of spread requires modeling networks of interactions. 
Denote the probability that polio will reemerge in country $i$ by $p_{i}$. There are two reasons polio might reemerge. There is a "residual" probability of reemergence, $\bar{p}$, in country $i$, which depends on the risks noted in section II-risks that are independent of whether country $i$ vaccinates with OPV in the post-eradication era. As noted, steps are being taken to lower this probability. While it is assumed here that the value of $\bar{p}$ is given, the analysis also shows how changes in this probability affect behavior in the vaccination cessation game. ${ }^{17}$ The costs of lowering these residual risks is not modeled.

There is an additional probability of reemergence, $\tilde{p}$, which depends on whether country $i$ vaccinates with OPV in the post-eradication age. Because this probability is independent of $\bar{p}$, the probability that polio will reemerge in $i$ in the post-eradication era depends on the sum of these probabilities minus their product. That is,

$$
p_{i}\left(q_{i}^{O}\right)=\bar{p}+\tilde{p} q_{i}^{O}-\bar{p} \tilde{p} q_{i}^{O}
$$

or

$$
p_{i}(0)=\bar{p}, p_{i}(1)=\hat{p}=\bar{p}+\tilde{p}(i-\bar{p}) .
$$

Given the assumption that countries are symmetric and that polio reemergence anywhere poses an equal threat to susceptible populations everywhere, the probability that polio will reemerge anywhere in the world $(p)$ will equal one minus the probability that polio will not reemerge anywhere. From the perspective of country $i$, this probability will look like,

$$
p=1-\left(1-p_{i}\right) \prod_{j \neq i}\left(1-p_{j}\right)
$$

Put differently, from the perspective of country $i$, the probability that polio will reemerge anywhere can be written $P_{i}\left(q_{i}^{\mathrm{O}} ; k\right)$, where $k$ denotes the number of other countries that vaccinate with OPV post-eradication. This gives

(8) $P_{i}(0 ; k)=1-(1-\bar{p})^{N-K}(1-\hat{p})^{k}, P_{i}(1 ; k)=1-(1-\bar{p})^{N-k-1}(1-\hat{p})^{k+1}$.

\section{Economics}

To derive the welfare consequences of vaccination cessation, some economic parameters need to be introduced. Let $b_{i}$ represent the monetary value of the welfare loss to country $i$ of a person in $i$ being paralyzed by polio (as noted in footnote 3, only about 1 in 200 people infected with poliovirus show

17. The returns to lowering this probability depend on the prospects of future vaccine cessation, just as the economics of vaccine cessation improve as this residual probability becomes smaller. 
symptoms of paralysis). The value of $b_{i}$ will be higher for a rich country than for a poor country, not because people in rich countries are worth any more as human beings but because their constraints and opportunities are different. ${ }^{18}$

Let $c_{i}^{O}$ denote the cost per individual immunized with OPV and $c_{i}^{I}$ the equivalent cost for IPV. As noted, the relative costs of OPV and IPV depends on the costs per dose and on the efficacy of the two vaccines in different environments. Relative costs also reflect the full costs, not only the manufacturing cost. The cost of OPV relative to IPV is high for rich countries, especially because of the possibility of vaccine-associated paralytic polio (as just noted, the welfare loss of a case of polio is higher for rich than for poor countries). It is primarily for this reason that rich countries have already switched to IPV. For poor countries, the costs of immunizing with OPV are low relative to the costs of IPV. This is not only because the welfare consequences of vaccine-associated paralytic polio are relatively low; it is also because IPV is more costly to produce and must be injected, requiring the assistance of trained health workers. Moreover, OPV is believed to provide greater population immunity in poor-sanitation environments. Poor countries have not switched to IPV. ${ }^{19}$

Pulling all of this together, country $i$ receives the following expected post-eradication payoff

$$
\begin{aligned}
\pi_{i}\left(q_{i}^{O}, q_{i}^{I} ; k\right)= & P_{i}\left(q_{i}^{O} ; k\right)\left\{-b_{i} \mu_{i} R_{0} v^{c}\left(1-q_{i}^{O}-q_{i}^{I}\right)\left[1-v^{c}\left(q_{i}^{O}+q_{i}^{I}\right)\right] n\right\} \\
& -\left(c_{i}^{O} q_{i}^{O}-c_{i}^{I} q_{i}^{I}\right) v^{c} n .
\end{aligned}
$$

Equation (9) assumes that countries are risk-neutral, a standard assumption that is approximately consistent with state behavior. If countries were risk averse, they would not even attempt to eradicate polio. If countries were risk loving, rich countries would not continue to vaccinate with IPV and poor countries would not be apprehensive about stopping OPV vaccination in the post-eradication era.

The most important point to note about equation (9) is that the payoff to every country $i$ depends not only on the choices made by $i$ but also on the choices made by other countries. Interdependence appears in the first term in equation (9), the probability that polio will remerge anywhere and thus

18. This is the same reason that the value of a statistical life is higher in rich than in poor countries (Viscusi and Aldy 2003). Note as well that the benefits of eradication can be broader than suggested by this value. Bleakley (2007) shows that the elimination of hookworm in the United States led to improvements in both literacy and income. Miguel and Kremer (2004) find that treatment for worms in Kenya improved health and school participation even among untreated children.

19. Thompson and others (2008) estimate the net benefits for rich and poor countries of using the two vaccines. Their analysis confirms the greater attractiveness of OPV in poor countries and of IPV in rich. It also quantifies the gains to ceasing routine immunization. For an empirical analysis demonstrating the economic advantage of OPV over IPV in South Africa, see Griffiths, Botham, and Schoub (2006). 
threaten $i$. This probability, as summarized in equation (8), depends on whether $i$ and other countries vaccinate with OPV. ${ }^{20}$

The term in curly brackets in equation (9) consists of three parts: $b_{i}$ denotes the social cost of an infection; $\mu_{i} R_{0} v^{c}\left(1-q_{i}^{O}-q_{i}^{I}\right)$ represents the probability that a randomly selected susceptible individual in $i$ will become infected; and $\left[1-v^{c}\left(q_{i}^{\mathrm{O}}+q_{i}^{I}\right)\right] n$ represents the number of such individuals $(n$ is total population size, assumed to be equal for all countries) in country $i .^{21}$ The entire term in curly brackets thus represents the welfare consequences for country $i$ of an outbreak. The value will equal zero if the country is unaffected by an outbreak, because it continues to vaccinate with either OPV or IPV at the level $v^{c}$; the value will be negative (that is, the country will suffer a loss) if the country stops vaccinating.

While vaccination to the level $v^{c}$ with either OPV or IPV can protect a country from possible reemergence, vaccination is costly. The last term in equation (9) represents the total cost of immunizing $i$ 's population, using OPV and IPV, respectively. Since differences in the efficacy of these vaccines are reflected in their costs (number of doses needed to stimulate immunity), country $i$, should it choose to vaccinate, can be expected to use the vaccine that supplies population immunity at lowest cost. Herein lies the global problem. Use of OPV by $i$ protects $i$ 's susceptible population but increases the probability of an outbreak globally in the period following certification. Similarly, use of OPV by any country $j, j \neq i$, increases the probability that $i$ will be vulnerable to an outbreak in the post-eradication era. It is because of such externalities that maximization of equation (9) by each country will likely not maximize the sum of the payoffs for all countries.

\section{AnALYSIS}

How can countries be expected to behave? And how will the behavior of individual countries affect the well-being of other countries and the world as a whole?

20. As expressed in equation (9), the probability of an outbreak does not depend on whether countries continue using IPV. According to Thompson and Duintjer Tebbens (2008), for a scenario of global coordination of vaccination/vaccination cessation, the expected number of post-eradication outbreaks is marginally lower for a poor country that vaccinates with IPV than for a poor country that does not vaccinate at all. For a rich country, the expected number of post-eradication outbreaks is marginally higher for a rich country that continues to use IPV than for one that does not vaccinate at all, because of the risks associated with IPV manufacture. Quantitatively, these differences are very small. The major differences in the expected number of outbreaks identified by Thompson and Duintjer Tebbens concern the use, or nonuse, of OPV. These differences are a central feature of equation (9).

21. In the absence of vaccination, the entire population is assumed to be susceptible. This is not the situation that will exist immediately after eradication has been certified. Throughout most of this article, this assumption does not matter because $n$ drops out of the important relationships. It will matter in the section on further calculations, but here separate calculations are made. 
From equation (9), the payoffs to country $i$ of every possible outcome are:

$$
\begin{gathered}
\pi_{i}(0,1 ; k)=-c_{i}^{I} v^{c} n \\
\pi_{i}(1,0 ; k)=-c_{i}^{O} v^{c} n \\
\pi_{i}(0,0 ; k)=-\left[1-(1-\bar{p})^{N-k}(1-\hat{p})^{k}\right] b_{i} \mu_{i} R_{0} v^{c} n .
\end{gathered}
$$

As in figure $1 \mathrm{~b}$, the payoff to each country $i$ of vaccinating (with OPV or IPV) is constant, while the payoff to $i$ of stopping vaccination depends on how many other countries vaccinate with OPV in the post-eradication era. But whereas figure $1 \mathrm{~b}$ was offered as a crude sketch, equations $(10 \mathrm{a}-\mathrm{c})$ are built on strong epidemiological and economic foundations and incorporate key parameters for analysis.

Recall that figure $1 \mathrm{~b}$ assumes that countries are symmetric. It is useful to begin by applying equations $(10 \mathrm{a}-\mathrm{c})$ to characterize all possible outcomes under this same assumption of symmetry before turning to the empirically relevant situation in which countries differ. That analysis will build on the previous one by assuming that all rich countries are alike and that all poor countries are alike. After analyzing a symmetric rich-country-only world and a symmetric poor-country-only world, these two worlds are combined to characterize the important policy situation facing us today-vaccination cessation in an asymmetric world.

\section{Symmetry}

In this model, it is easy to see that if countries are symmetric they will behave symmetrically in equilibrium. Three symmetric outcomes are possible. All countries will vaccinate with OPV, all countries will vaccinate with IPV (and cease vaccinating with OPV), or all countries will cease vaccinating altogether. Which of these outcomes can be sustained as equilibria of the polio post-eradication game? As table 1 shows, there are five cases, depending on parameter values. These are illustrated in figures $2 \mathrm{a}-\mathrm{e}$.

For cases I-III, countries that vaccinate prefer OPV to IPV. In case I, global OPV cessation is better for every country $i$ than continued use of OPV, but each country is better off ceasing to vaccinate with OPV only if $\bar{k}$ or fewer other countries continue to vaccinate with OPV. If more than that number of countries continue to vaccinate with OPV, the risk of cVDPV emergence is so great that every country prefers to vaccinate with OPV. In case I, therefore, there are two equilibria (denoted by the closed dots in figure $2 \mathrm{a}$ ), one in which every country vaccinates with OPV and one in which no country vaccinates for polio. ${ }^{22}$ OPV cessation is thus characterized by tipping, and $\bar{k}$ is the "tipping point." (If and

22. These equilibria are in pure strategies. There is also a symmetric equilibrium in mixed strategies corresponding to case I, in which each country chooses to vaccinate with OPV (as opposed to not vaccinating at all) according to a certain probability distribution; this equilibrium is unstable. 
Barrett 373

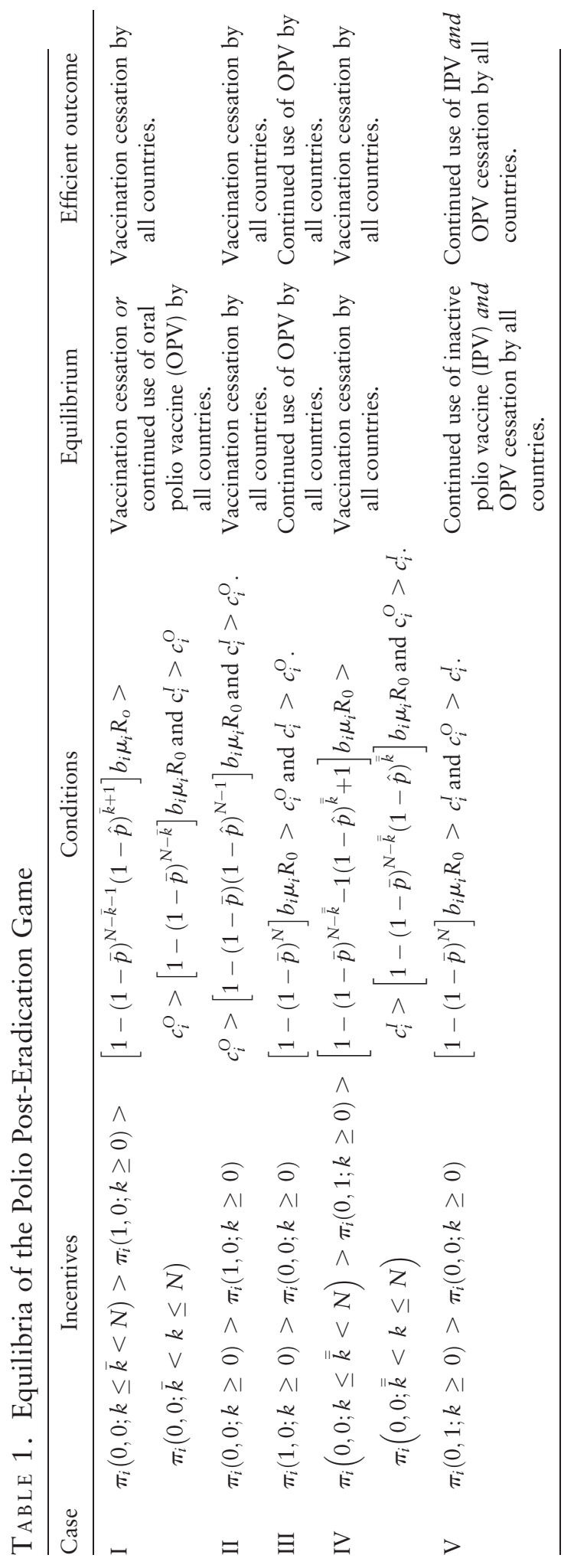



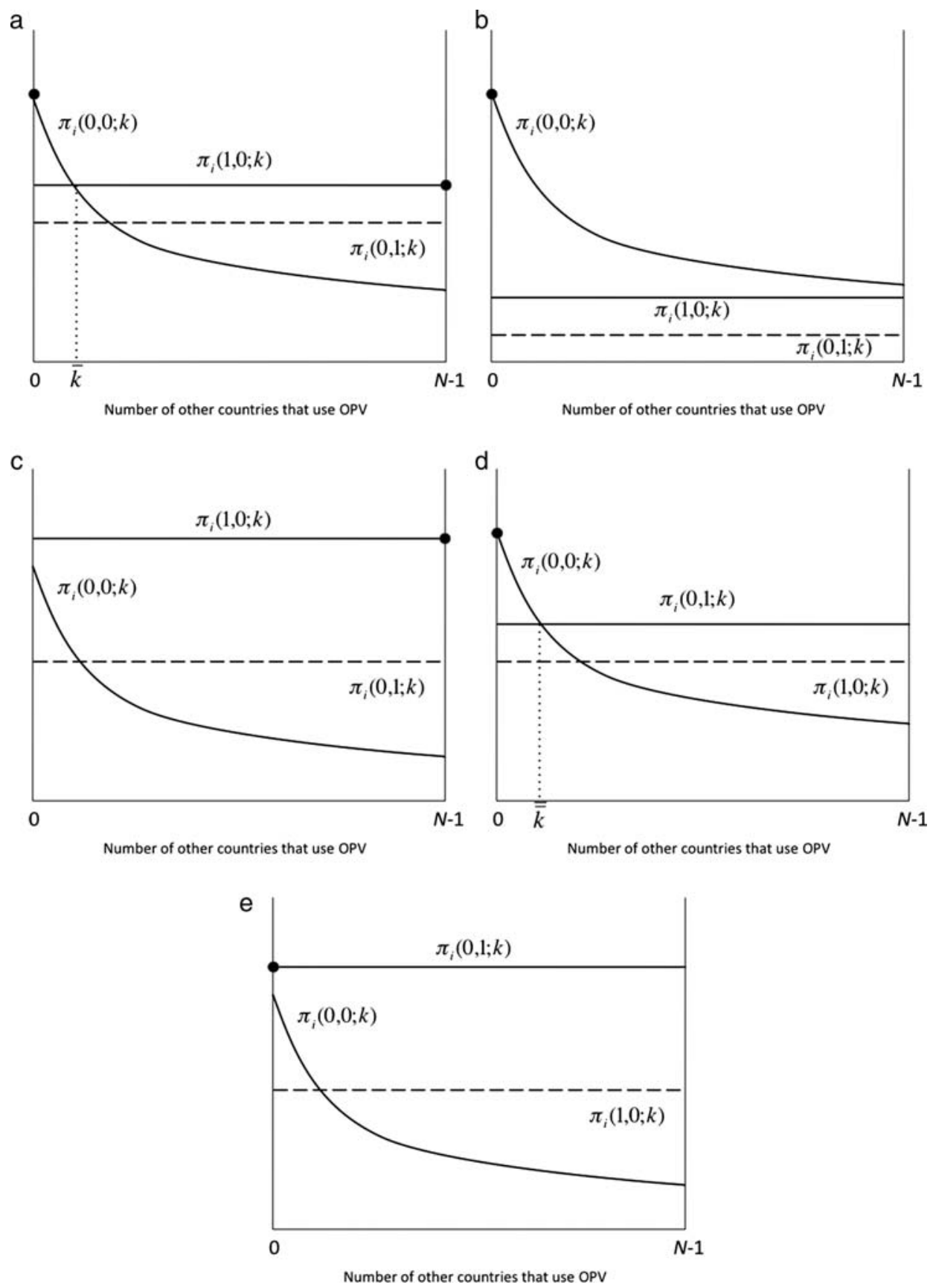

Figure 2. Symmetric Vaccination Cessation Games

only if $\bar{k}=0$ is universal coordination needed.) Coordination is required to sustain the efficient equilibrium in which there is a vaccination cessation dividend; how this can be achieved is discussed in the next subsection. 
For cases II and III, coordination is not required. In case II, each country prefers vaccination cessation to use of OPV irrespective of whether any country vaccinates with OPV (figure $2 \mathrm{~b}$ ). In case III (figure $2 \mathrm{c}$ ), every country is better off vaccinating with OPV, whether others do or not.

Which case is supported depends on the parameter values. Clearly, for case I to hold, the payoff to vaccination cessation must fall with the number of other countries that continue to vaccinate with OPV. As shown in figure $3 \mathrm{a}$, the OPV cessation payoff curve will be pulled down (relative to the right hand axis) and in (relative to the left hand axis) as the probability of reemergence due only to continued OPV vaccination $(\tilde{p})$ increases. Of course, for case I to hold, it is also essential that the value of this payoff be high when all countries cease vaccinating; figure $3 \mathrm{~b}$ shows that this requires that the residual probability of reemergence be kept low. As explained in section II, this is precisely what the WHO is working towards.

Relying again on figures $2 \mathrm{a}$ and $\mathrm{b}$, it is easy to see that case II will be supported only if the residual probability of reemergence and the probability of a cVDPV outbreak are both very small. By contrast, figure $2 \mathrm{c}$ shows that case III will be supported only if the residual probability is very high (it will not matter in this case whether the probability of a cVDPV is high or low).

For cases IV and V, countries that vaccinate prefer IPV to OPV. In case IV (figure $2 \mathrm{~d}$ ), every country prefers not to vaccinate at all provided $\overline{\bar{k}}$ or fewer other countries vaccinate with OPV. Since, by assumption, no country prefers OPV to IPV, vaccination cessation is the unique equilibrium. In case $\mathrm{V}$ (figure 2e), by contrast, every country is better off vaccinating with IPV, irrespective of what other countries do. To sustain the equilibrium in case IV, the residual probability of reemergence must be very low. To sustain the equilibrium in case $\mathrm{V}$, the residual probability of reemergence must be high. In both cases, the probability of cVDPV emergence is irrelevant, since countries will not use OPV under any circumstances.

Table 2 summarizes these five cases. ${ }^{23}$ Two main conclusions emerge. First, for countries to reap a surplus from eradication, vaccine use must cease, an outcome that can be sustained only for cases I, II, and IV (in the other cases, vaccination cessation is inefficient). As is clear from the table, vaccination cessation depends on the residual risk of reemergence being low. Second, vaccination cessation is an "international problem" only for case I. Only this case requires coordination to sustain vaccination cessation. For cases II and IV, vaccination cessation is a dominant strategy.

23. The table ignores parameter $b$, the monetary value of the welfare loss of a person being paralyzed by polio. Plainly, so long as the probability of reemergence is nonzero, $b$ must not be "too high" in order for vaccination cessation to yield positive returns. 
Figure 3. Shapes of Vaccination Cessation Payoff Curves
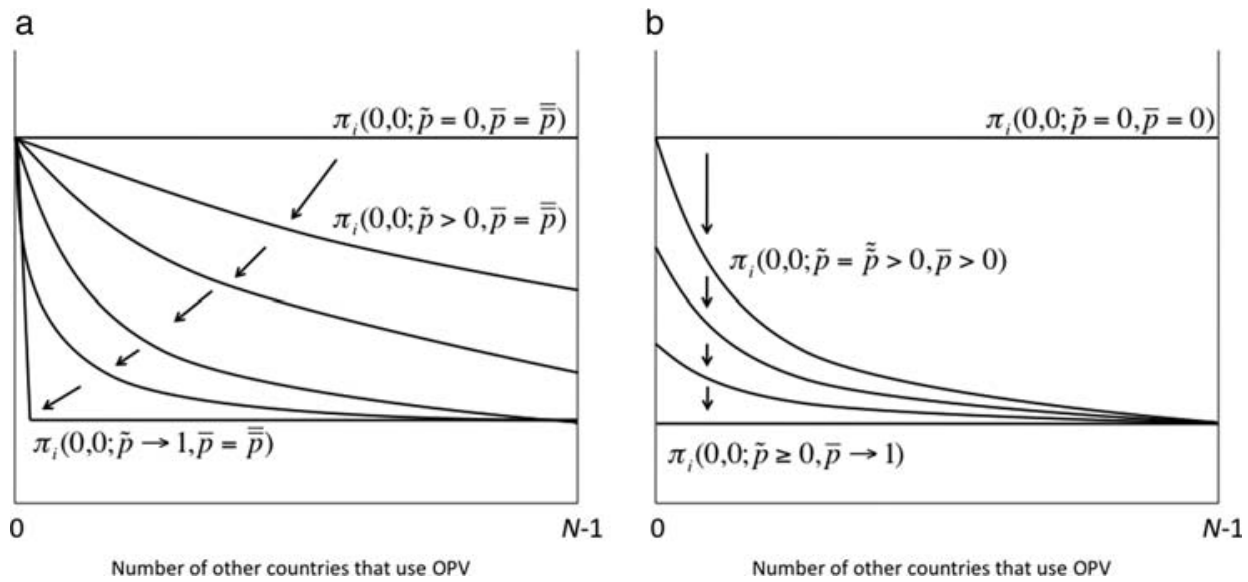

Table 2. Implications of Parameter Value Combinations

\begin{tabular}{|c|c|c|c|c|c|}
\hline Case & $c_{i}^{\mathrm{O}} / c_{i}^{I}$ & $\bar{p}$ & $\tilde{p}$ & $\begin{array}{l}\text { Positive } \\
\text { returns to } \\
\text { eradication }\end{array}$ & $\begin{array}{l}\text { Coordination } \\
\text { required to } \\
\text { claim these } \\
\text { returns? }\end{array}$ \\
\hline I & Low & Low & High & Yes & Yes \\
\hline II & Low & Low & Low & Yes & No \\
\hline III & Low & High & NA & No & - \\
\hline IV & High & Low & NA & Yes & No \\
\hline V & High & High & NA & No & - \\
\hline
\end{tabular}

- No returns to eradication, and so no reason to coordinate.

\section{Coordination}

If coordination is required for case I, how can it be achieved? It is unlikely that countries can coordinate vaccination cessation unaided, particularly as they are starting from a situation in which all countries are vaccinating (the status quo thus favors continued vaccination). It is also unlikely that a recommendation by the WHO will suffice. What each country requires is an assurance from all the other countries that they intend to stop vaccinating. ${ }^{24}$ This could be achieved by "a World Health Assembly resolution

24. Technically, if the tipping point were greater than zero, countries would be willing to stop vaccinating even if one or a few countries refused to stop. If the tipping point were very large, coordination might be achieved by a small number of countries moving first (by stopping OPV vaccination), as in a Stackelberg model. For the problem at hand, a very large number of countries must stop for it to be attractive for the others to stop. 
outlining the precise timing and process for simultaneous OPV cessation by all OPV-using countries" WHO (2005, p. 8).

World Health Assembly resolutions are nonbinding. Fortunately, a stronger institution is not needed to coordinate vaccination cessation. ${ }^{25}$ All that is needed is that every country vote in favor of a resolution obligating every country to stop vaccinating with OPV. Reputational matters aside, a country that intends to vaccinate with OPV, whatever other countries do, cannot gain by deceiving the other members of the WHO. So communication of intentions here will be taken seriously and will change how countries behave.

\section{Back-of-the-Envelope Calculations}

What does the game look like in the "real world"? This is an asymmetric world, and play will depend on the values of the key model parameters. Here, the values shown in table 3 are used, most of them taken from Thompson and Duintjer Tebbens (2008). ${ }^{26}$ These values treat all poor countries as alike, and all rich countries as alike but distinguish between rich and poor countries. The calculations made here and later in this article should be interpreted as a general indication of the incentives that states have to stop vaccinating. ${ }^{27}$ It should be obvious that the model is deliberately simple and ignores issues that may require further analysis (such as whether immunity in poor countries can be sustained without supplementary immunization activities). These rather obvious qualifications aside, however, the estimates presented here point to some powerful policy conclusions.

The change over time in the residual probability of an outbreak in poor countries is particularly important. In rich countries, which stopped vaccinating with OPV years ago, the probability of cVDPVs and iVDPVs emerging will be very low after eradication has been certified. By contrast, in poor countries, the residual probability will be high immediately after OPV cessation, but it will decline to about the same level as in rich countries within a few years. The discussion therefore distinguishes between the consequences of OPV cessation in the short and long term.

25. A resolution adopted by the World Health Assembly in 2008 instructed the WHO to develop "a mechanism to mitigate the risk of reintroduction of poliovirus that does not involve amending the International Health Regulations or developing another binding instrument [emphasis added]" (WHO 2008). An amendment to the International Health Regulations would be binding on parties but has been ruled out. Fortunately, it is not needed.

26. As a quick check on the plausibility of the probability estimates, note that Duintjer Tebbens and others $(2006$, p. 1478-79) say that the probability of at least one outbreak globally is 60-95 percent in the first year after vaccination cessation and 1-6 percent at the end of the third year. The values in table 3 imply a probability of 84 percent in the first year and 1 percent after three years-a close correspondence.

27. Note especially that the probability estimates in Thompson and Duintjer Tebbens (2008) assume coordinated action by all countries. The current article assumes that the same probabilities apply when polices are less than fully coordinated. 
Table 3. Parameter Values for Quantitative Analysis

\begin{tabular}{lcc}
\hline Parameter & Poor countries & Rich countries \\
\hline $\bar{p}(t=0), \bar{p}(t=3)$ & $0.03,0.0001$ & $0.0001,0.0001$ \\
$\hat{p}$ & 0.06 & 10 \\
$N$ & 60 & 0.0125 \\
$\mu_{i}$ & 0.0175 & 6 \\
$R_{O}$ & 6 & - \\
$c_{i}^{O}$ & $\$ 2.38$ & $\$ 38.78$ \\
$c^{I}$ & $\$ 4.72$ & $\$ 1,700$ \\
$b_{i}$ & $\$ 26.50$ & \\
\hline
\end{tabular}

Note and source: The values for $\bar{p}$ are approximations of the values derived by Thompson and Duintjer Tebbens (2008; hereafter, TDT) and are for two periods. The values represent the probability of at least one outbreak in the first year following certification of eradication of the wild viruses $(t=0)$ and three years after this date $(t=3)$. The values reflect a scenario in which the inactive polio vaccine (IPV) is used without any supplemental immunization prior to certification, for circulating vaccine-derived polioviruses (cVDPVs) only, assuming realistic population immunity and containment of wild viruses. These values are virtually identical for a scenario of no routine vaccination. The values are calculated using estimates of the Poisson rates for an outbreak (available at http://www.kidrisk.org). If this rate is represented by $\sigma$, then the probability of an outbreak is given by $1-e^{-\sigma}$, where $e^{-\sigma}$ is the probability of there being no outbreaks and $1-e^{-\sigma}$ is therefore the probability of there being at least one outbreak in a year.

The estimate for $\hat{p}$ is taken from the same source and is for the same scenario except that oral polio vaccine (OPV) is used instead of IPV or no vaccination. This probability is constant over time. The values for $\tilde{p}$ are not estimated separately by TDT but can be obtained by substituting the values for $\hat{p}$ and $\bar{p}$ in equation (6).

TDT calculate probabilities for representative countries with a population of 100 million. There are nearly 7 billion people in the world, with just over 1 billion in rich countries and nearly 6 billion in developing countries, including upper middle-income countries. The values for $\mathrm{N}$ assumed here are rough approximations.

The parameter $\mu$ can be approximated by $1 / L$, where $L$ is life expectancy in years (see Anderson and May 1991, p. 66). Life expectancy of 57 years in poor countries implies $\mu_{P}=$ 0.0175 , and 79 years in rich countries, $\mu_{R}=0.0125$.

A value of $R_{0}=6$ implies a critical vaccination level of 83 percent. The value may be twice as large in some developing countries with high population densities and poor sanitation (Thompson and others 2008; Fine and Ritchie 2006). Sensitivity analysis is presented in section III.

Estimates of the costs of immunization and of the benefits of an avoided case of paralytic polio are from Thompson and others (2008) and Duintjer Tebbens, Sangrujee, and Thompson (2006). These analyses assume that OPV costs $\$ 0.10$ per dose, three doses are needed for immunity, the vaccine is subject to 20 percent wastage, and the nonvaccine cost is $\$ 2$ per immunized child in poor countries. In rare cases, OPV causes vaccine-associated paralytic polio. This cost is excluded from the estimates in the table. IPV is assumed to cost $\$ 1$ per dose in poor countries, with a 10 percent wastage rate, two doses required for immunity, and a nonvaccine cost of $\$ 2.50$ per fully immunized child. In rich countries, IPV is assumed to cost $\$ 10$ per dose, with a 5 percent wastage rate, three doses required for full immunity, and a nonvaccine cost of $\$ 7.20$ for a combination vaccine. Note that $b_{i}$ represents the value of an avoided infection. Since only one in 200 infected people show paralytic symptoms, the values from Thompson and others (2008) of an avoided case of paralytic polio are divided by 200 to obtain the values in the table.

To begin, it helps to consider two hypothetical worlds, one comprising only poor countries and the other comprising only rich countries. The more realistic mixed situation is considered in the next subsection. 
Analysis of a poor-country-only world and a rich-country-only world can rely on the symmetric model summarized in tables 1 and 2 . Using the values in table 3 for poor countries results in, $\left[1-(1-\bar{p})^{N-\bar{k}-1}(1-\hat{p})^{\bar{k}+1}\right] b_{i} \mu_{i} R_{0}>c_{i}^{o}>$

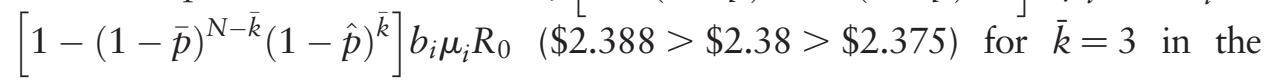
first year after vaccination cessation. In the short run, then, a world made up of only poor countries is a case I world. After three years, $c_{i}^{o}>\left[1-(1-\bar{p})(1-p)^{N-1}\right] b_{i} \mu_{i} R_{0}(\$ 2.38>\$ 0.02)$. Thus, in the longer run, a world made up of only poor countries is a case II world. All poor countries will want to stop vaccinating in the longer run, but coordination of vaccination cessation is needed in the short run.

For rich countries, the residual probability in table 3 is constant over time, and so in a world made up only of rich countries, there is nothing to distinguish the short from the longer run. Using the values in table 3 yields $\left[1-(1-\bar{p})^{N}\right] b_{R} \mu_{R} R_{0}=\$ 0.13$, which is substantially less than the cost of vaccinating with IPV (\$38.78). Thus, the values in table 3 imply that a world of only rich countries would be a case IV world. Rich countries would want to stop vaccinating with IPV immediately after certification of eradication.

\section{Asymmetry}

This section combines the world of rich countries and that of poor countries.

If $\bar{p}$ and $\tilde{p}$ were both low, all countries would unilaterally cease vaccinating in the post-eradication era, even though rich and poor countries would be asymmetric. If $\bar{p}$ were high, by contrast, it wouldn't matter whether $\tilde{p}$ were high or low; all countries would continue to vaccinate in the post-eradication era (with the poor using OPV and the rich IPV). Here, the focus is on the last and most interesting asymmetric case, that with $\bar{p}$ low and $\tilde{p}$ high-the case the world is most likely to be facing today (see table 3 ) and the case the WHO is planning for.

Rich countries have already switched to IPV and have no incentive to use OPV for routine immunization. Using the values in table 3, it is easy to confirm that the probability of a global outbreak originating in rich countries is so small as to have no bearing on the decision by poor countries to stop vaccinating. In an asymmetric world, poor countries will thus act in the same way as described in the previous subsection. In the immediate post-eradication era, poor countries will either continue to vaccinate with OPV or they will cease vaccinating (figure $4 \mathrm{a}$ ). If poor countries can coordinate vaccination cessation in the short run, their incentives not to vaccinate will strengthen over time (this is from an ex ante perspective; see the concluding section of this article for the implications of that perspective).

The behavior of rich countries, by contrast, will depend on what poor countries do. Letting subscripts $R$ and $P$ denote rich and poor countries, with 
$N_{R}+N_{P}=N$, the payoffs to rich countries can be written as:

$$
\begin{gathered}
\pi_{R}\left(0,0 ; k_{p}=0 ; k_{R}\right)=-\left[1-(1-\bar{p})^{N-k_{R}}(1-\hat{p})^{k_{R}}\right] b_{R} \mu_{R} R_{0} v^{c} n \\
\pi_{R}\left(0,0 ; k_{p}=N_{P} ; k_{R}\right)=-\left[1-(1-\bar{p})^{N_{R}-k_{R}}(1-\hat{p})^{N_{P}+K_{R}}\right] b_{R} \mu_{R} R_{0} v^{c} n .
\end{gathered}
$$

Of course, rich countries will not use OPV in the post-eradication era; thus, $k_{R}=0$. Using the values in table 3 yields $\left[1-\left(1-\bar{p}_{R}\right)^{N_{R}}\left(1-\bar{p}_{P}\right)^{N_{P}}\right] b_{R} \mu_{R} R_{0}=\$ 107.02$ for the period immediately following certification and $\$ 0.89$ three years later. Since the cost of vaccinating with IPV (\$38.78) lies between these values, if poor countries stop vaccinating, rich countries will also want to stop vaccinating, but only in the longer run (figure 4b). Finally, $\left[1-\left(1-\bar{p}_{R}\right)^{N_{R}}\left(1-\hat{p}_{P}\right)^{N_{P}}\right] b_{R} \mu_{R} R_{0}=\$ 124.39$, which exceeds the cost of vaccinating with IPV (\$38.78). If poor countries fail to coordinate vaccination cessation, rich countries will continue to vaccinate with IPV.

In an asymmetric world, rich and poor countries are participants in a coupled coordination game. As indicated in figure 4, the game has two equilibria. In one, poor countries continue to vaccinate with OPV, and rich countries with IPV. In the other, poor countries stop vaccinating with OPV and, after a time, rich countries stop vaccinating with IPV. Only this second equilibrium is efficient.

Figure 4. Payoffs to Poor and Rich Countries in the Asymmetric Game
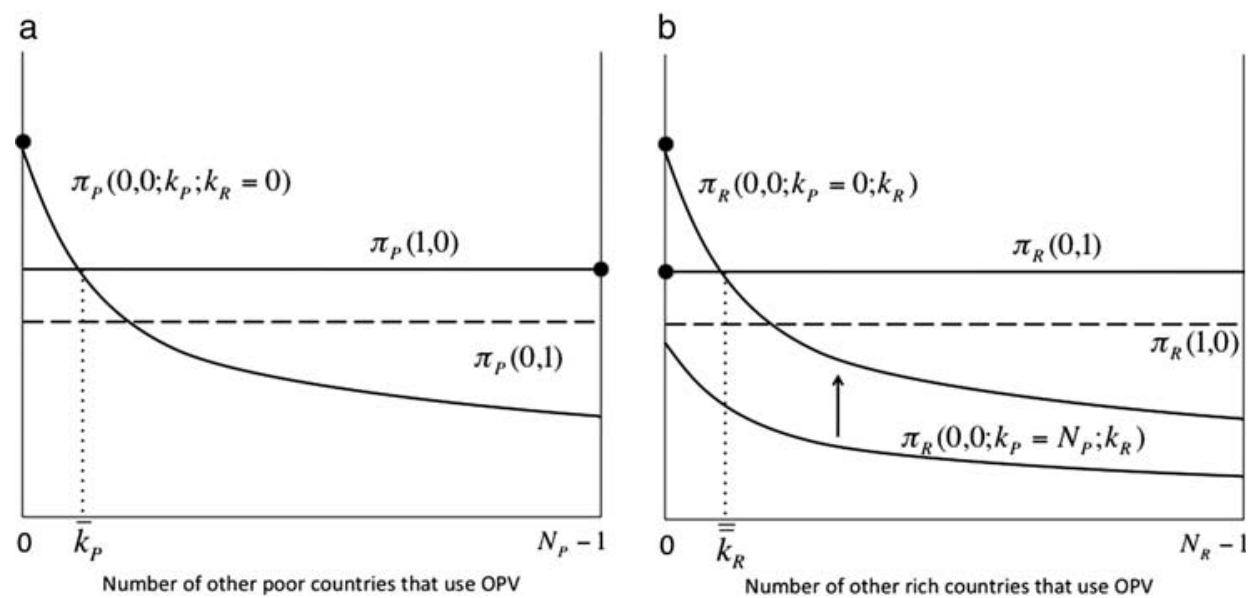


\section{Sensitivity Analysis}

Before examining the coupled coordination game in more detail, consider briefly the sensitivity of the results obtained thus far. In particular, consider the sensitivity to one of the parameters for rich countries and to another one for poor countries.

In table 3, the value of an avoided case of paralytic polio in rich countries is assumed to be $\$ 340,000$ (in table 3, this value is divided by 200 to obtain an average value per case of infection, as described in the table notes). Analysis by Miller and others (1996), however, suggests that the U.S. decision to switch to IPV is consistent with a value of up to $\$ 11.2$ million. Will use of this larger value change the conclusions reached above? It is easy to show that, in the long run, provided that all poor countries stop using OPV, use of this higher value raises the value of $\left[1-\left(1-\bar{p}_{R}\right)^{N_{R}}\left(1-\bar{p}_{P}\right)^{N_{P}}\right] b_{R} \mu_{R} R_{0}$ from $\$ 0.89$ (as shown above) to $\$ 25.13$. Although much larger, the value is still smaller than the cost of vaccinating with IPV (\$38.78). Thus, the conclusion that rich countries will want to stop vaccinating with IPV in the longer run, provided poor countries stop vaccinating with OPV, seems robust with respect to this parameter.

In some poor countries, as noted, the value of $R_{0}$ may be higher than indicated in table 3. If, for example, $R_{0}=12$ in poor countries, then $\left[1-\left(1-\bar{p}_{R}\right)^{N_{R}}\left(1-\bar{p}_{P}\right)^{N_{P}}\right] b_{P} \mu_{P} R_{0}=\$ 4.67$, which is greater than $c_{i}^{o}=\$ 2.38$, implying that poor countries will want to continue to vaccinate with OPV in the immediate post-eradication era. This is a very different result than obtained previously. However, if poor countries stop vaccinating in the immediate post-eradication era, in the longer run $\bar{p}$ will fall to 0.0001 for the countries that stop vaccinating. It can be shown that each poor country will want to stop vaccinating with OPV in the longer run provided that no more than eight other countries continue to vaccinate with OPV. A higher value for $R_{0}$ thus reduces the incentives for poor countries to stop vaccinating. However, poor countries will still have an incentive to coordinate OPV cessation provided they do not discount the future too heavily.

\section{Further Calculations}

Though rich and poor countries can both gain from coordination, the relationship is decidedly asymmetric. Rich countries will benefit, without bearing any of the risk, if the poor stop vaccinating with OPV. In the period immediately following OPV cessation, susceptible people in poor countries will be vulnerable to outbreaks. In rich countries, by contrast, everyone will be protected by IPV. It seems plausible that the poor countries will therefore condition their cessation of OPV on assistance being given by the rich countries. Poor countries may reason that the burdens of OPV cessation ought to be shared since the benefits will be. Considerations of equity favor universal vaccination with IPV for a period immediately following certification. 
Consider some crude calculations. If at the time eradication of the wild viruses is certified, vaccination has been maintained at a level that guarantees herd immunity everywhere, the differential risk of OPV cessation to poor (relative to rich) countries can be eliminated by having poor countries substitute IPV for OPV for about three years. Unlike immediate vaccination cessation, such a policy will protect susceptible newborns. Assuming a population of 6 billion people in poor countries and a birth rate of 1.75 percent, there will be 105 million newborns in poor countries in the first year after certification. To maintain herd immunity assuming $R_{0}=6,83$ percent of newborns must be vaccinated. At an additional cost per person vaccinated of $\$ 2.34\left(c_{P}^{I}-c_{P}^{O}=\$ 4.72-\$ 2.38=\$ 2.34\right)$, the annual cost of substituting IPV for OPV will be about $\$ 200$ million. Assuming $R_{O}=12$, 92 percent of newborns must be vaccinated, raising the costs of IPV substitution to $\$ 225$ million. Based on an independent and more detailed analysis, and assuming three doses of IPV per person vaccinated (the above analysis assumes two doses in poor countries), Liu and others (2003) put the cost at $\$ 317$ million. $^{28}$ These are relatively small numbers-less than half the amount being spent now to achieve eradication.

What are the benefits to rich countries? If poor countries cease vaccinating with OPV, the analysis here shows that rich countries will prefer not to vaccinate with IPV once the residual risk of an outbreak has fallen to a very low level. Rich countries will save \$39 for every vaccination avoided. Not vaccinating means that susceptible people will be exposed to the risk of an outbreak, but the expected cost is just $\$ 0.89$ per person. Once the residual probability has fallen to a very low level, vaccination cessation might save rich countries $\$ 400$ million in the first year. ${ }^{29}$ At the higher risk cost considered in the previous subsection, the saving would shrink to about $\$ 140$ million in the first year-still a substantial saving. ${ }^{30}$ Moreover, these savings will grow over time. It is thus plain that rich countries can very quickly recoup the costs of subsidizing IPV use in poor countries for about three years. ${ }^{31}$ Poor countries will also

28. Assuming three doses, the additional cost of IPV over OPV, by my calculations, would be between about $\$ 300$ million (for $R_{0}=6$ ) and $\$ 335$ million (for $R_{0}=12$ ). The estimate by Liu and others (2003) lies in the middle of this range.

29. Multiply the savings per person $(\$ 38.78-\$ 0.89$, or $\$ 37.89)$ by the number of people. The latter is found by multiplying the population level in rich countries (1 billion) by the birth rate $(.0125)$ and then multiplying this figure by the critical vaccination level (0.8333). That yields a savings in the first year of about $\$ 395$ million.

30. The savings per person would then be $\$ 38.78-\$ 25.13$, or $\$ 13.65$. The estimate given here is found by using this value in the calculation described in the previous footnote.

31. Consider a rough calculation. Assume that substitution costs $\$ 317$ million a year and lasts three years. The total cost would be $\$ 951$ million. Assume further that this must be paid up front. Then assume that vaccination cessation by rich countries is valued at $\$ 140$ million a year. If poor countries will stop vaccinating only if they are given the IPV subsidy, it will pay rich countries to offer the one-time subsidy provided $\$ 140 \mathrm{~m} / r>\$ 951$ million, where $r$ is the rate of discount, or $r<15$ percent. 
gain from this policy. Vaccination cessation is thus economically efficient and can be made to be incentive compatible. ${ }^{32}$

Rather than subsidize substitution of IPV, rich countries could lower the cost of IPV by financing research and development that will lead to a cheaper IPV vaccine. The WHO is currently exploring ways to do this-by fractional dosing (stimulating an immune response using less than the current recommended dose), reduced dosing (changing the current vaccination schedule by administering the vaccine to children at different ages), adding adjuvants (agents that increase the immune response, allowing the viral content in IPV to be reduced), and improving IPV process manufacture. Success in these areas will improve the economics of a (temporary) switch to IPV. It may even obviate the need for a subsidy. Of course, such an investment would shrink the gains to eventual vaccination cessation, but it would at the same time lower the cost of protecting susceptible populations from future, post-eradication risks.

\section{Concluding Comments}

Eradication is worth attempting only if it promises the world a return on investment, measured as avoided infections and vaccination costs. For smallpox, each country could claim its share of the return very easily after eradication was certified by deciding independently to stop vaccinating. For polio, vaccination cessation entails risks as well as rewards-and not only for the country choosing to stop vaccinating but for other countries as well. The post-eradication risks can be reduced, but only if countries coordinate their vaccination cessation decisions and make further investments (such as by substituting IPV for OPV, for a period of time).

Though this article has focused on the challenge of OPV cessation, the other risks to vaccination cessation have been accounted for in the analysis through the term called the "residual" probability of an outbreak. This probability was taken as given-it was not solved for-but of course these other risks can also be managed. Clearly, there will be tradeoffs in limiting these risks, just as there are in limiting the risks of OPV vaccination. One important insight from this article is that individual risks need to be evaluated jointly. As shown, investments that lower the residual risk may increase the returns to OPV cessation, thus making it more likely that countries will be able to earn a return on eradication.

32. Formally, this could be modeled as a sequential game, with rich countries collectively deciding whether to finance a time-limited switch to IPV by poor countries in stage 1 and with poor countries collectively deciding whether to continue to vaccinate with OPV or to stop vaccinating (after the time limit on the IPV subsidy ends) in stage 2. Note also that rich country financing of the time-limited switch to IPV by poor countries is another collective action problem. Each rich country would prefer that the others pay this cost. However, there are features of the financing game that should favor cooperation. For an analysis of the financing of smallpox eradication, see Barrett (2006). 
It is important to underscore the simplicity of this analysis. It assumed, for example, that IPV production can be scaled up very easily, despite current capacity constraints, and without increasing the residual risks of IPV manufacture. It also assumed that high vaccine coverage can be maintained in poor country environments without great effort. This is hard enough to do with OPV. It will be even harder with IPV, which must be injected. A more detailed analysis is needed to explore these and other post-eradication challenges.

The article has also taken only an ex ante perspective, focusing on the planning stage. After decisions are made (to stop vaccinating with OPV, to continue to vaccinate with IPV, and so on), the consequences will be revealed-an outbreak will occur or it will not occur; lives will be affected; payoffs will change. If an outbreak occurs, other steps will be taken. The epidemic will have to be controlled; eradication will need to be attempted again; the decision to stop vaccination will have to be revisited. If the underlying model and its parameter values remain unchanged, the analysis of what to do the next time around will remain the same. It is possible, however, that we will learn from the experience and make further adjustments or that the underlying situation will change for some other, unexpected reason. The game may be different the next time around.

Finally, this article has presumed that eradication of the wild viruses will succeed. As explained in section I, the incentives to eradicate polio are closely linked to the post-eradication game. If countries believe that vaccination will continue in the post-eradication period, the economics of eradication will appear unfavorable, and countries will not try to eradicate the wild viruses. That is why the results of this article are important even at this pre-eradication stage. Eradication may fail despite our best efforts, but we should not let it fail for lack of a strategy for creating incentives for countries to stop vaccinating in the post-eradication era.

\section{References}

Anderson, R.M., and R.M. May. 1991. Infectious Diseases in Humans: Dynamics and Control. Oxford, UK: Oxford University Press.

Aylward, R.B., and S.L. Cochi. 2004. "Framework for Evaluating the Risks of Paralytic Poliomyelitis after Global Interruption of Wild Poliovirus Transmission." Bulletin of the World Health Organization 82 (1): 40-46.

Aylward, R.B., R.W. Sutter, and D.L. Heymann. 2005. "OPV Cessation-The Final Step to a 'Polio-Free' World.” Science 310 (5748): 625-26.

Barrett, S. 2003. "Global Disease Eradication.” Journal of the European Economics Association 1 (2-3): 591-600.

—. 2006. "The Smallpox Eradication Game." Public Choice 130 (1): 179-207.

2007. Why Cooperate? The Incentive to Supply Global Public Goods. Oxford, UK: Oxford University Press.

- 2009. "Polio Eradication: Strengthening the Weakest Links." Health Affairs 28 (4): 1079-90.

Barrett, S., and M. Hoel. 2007. "Optimal Disease Eradication." Environment and Development Economics 12 (5): 627-52. 
Bleakley, H. 2007. "Disease and Development: Evidence from Hookworm Eradication in the American South.” Quarterly Journal of Economics 122 (1): 73-117.

Duintjer Tebbens, R.J., M.A. Pallansch, O.M. Kew, V.M. Cáceres, H. Jafari, S.L. Cochi, R.W. Sutter, R.B. Aylward, and K.M. Thompson. 2006. "Risks of Paralytic Disease Due to Wild or Vaccine-Derived Poliovirus after Eradication.” Risk Analysis 26 (6): 1471-1505.

Duintjer Tebbens, R.J., N. Sangrujee, and K.M. Tompson. 2006. "The Costs of Future Polio Risk Management Policies." Risk Analysis 26 (6): 1507-31.

Fine, P.E.M., and S. Ritchie, 2006. "Perspective: Determinants of the Severity of Poliovirus Outbreaks in the Post Eradication Era." Risk Analysis 26 (6): 1533-40.

Geoffard, P.Y., and T. Philipson. 1997. "Disease Eradication: Private versus Public Vaccination." American Economic Review 87 (1): 222-30.

Griffiths, U.K., L. Botham, and B.D. Schoub. 2006. "The Cost-Effectiveness of Alternative Polio Immunization Policies in South Africa." Vaccine 24 (29): 5670-78.

Kew, O.M., R.W. Sutter, E.M. de Gourville, W.R. Dowdle, and M.A. Pallansch. 2005. "Vaccine-Derived Polioviruses and the Endgame Strategy for Global Polio Eradication." Annual Review of Microbiology 59: 587-635.

Liu, X., A. Levin, M. Makinen, and J. Day. 2003. OPV vs IPV: Past and Future Choice of Vaccine in the Global Polio Eradication Program. Bethesda, MD: The Partners for Health Reformplus Project, Abt Associates, Inc.

Miguel, E., and M. Kremer. 2004. "Worms: Identifying Impacts on Education and Health in the Presence of Treatment Externalities.” Econometrica 72 (1): 159-217.

Miller, M., S. Barrett, and D.A. Henderson. 2006. "Control and Eradication." In Disease Control Priorities in Developing Countries, 2nd edition, ed. Dean T. Jamison, Joel G. Breman, Anthony R. Measham, George Alleyne, Mariam Claeson, David B. Evans, Prabhat Jha, Anne Mills, and Philip Musgrove. Oxford, UK: Oxford University Press.

Miller, M.A., R.W. Sutter, P.M. Strebel, and S.C. Hadler. 1996. "Cost-Effectiveness of Incorporating Inactivated Poliovirus Vaccine into the Routine Childhood Immunization Schedule.” Journal of the American Medical Association 276 (12): 967-71.

Thompson, K.M., and R.J. Duintjer Tebbens. 2007. "Eradication Versus Control for Poliomyelitis: An Economic Analysis.” The Lancet 369 (9570): 1363-71.

- 2008. "The Case for Cooperation in Managing and Maintaining the End of Poliomyelitis: Stockpile Needs and Coordinated OPV Cessation.” Medscape Journal of Medicine 10 (8): 190.

Thompson, K.M., R.J. Duintjer Tebbens, M.A. Palansch, O.M. Kew, R.W. Sutter, R.B. Aylward, M. Watkins, , Jr.H.E. Gary, J. Alexander, H. Jafari, and S.L. Cochi. 2008. "The Risks, Costs, and Benefits of Possible Future Global Policies for Managing Polioviruses.” American Journal of Public Health 98 (7): 1322-30.

Viscusi, W.K., and J.E. Aldy. 2003. "The Value of a Statistical Life: A Critical Review of Market Estimates Throughout the World." Journal of Risk and Uncertainty 27 (2): 5-76.

WHO (World Health Organization). 2005. "Framework for National Policy Makers in OPV-Using Countries." WHO/POLIO/05.02. www.polioeradication.org/content/publications/opvcessationframe workenglish.pdf.

. 2008. Sixty-first World Health Assembly Resolutions and Decisions. "Poliomyelitis: Mechanism for Management of Potential Risks to Eradication,” Agenda Item 11.2, WHA61.1. 19-24 May 2008, Geneva. www.polioeradication.org/content/general/WHA61_Resolution_English.pdf. 